\title{
Bei frühem Diabetesbeginn sinkt Lebenserwartung um über ein Jahrzehnt
}

\begin{abstract}
Die Diabetesepidemie scheint nicht aufzuhalten. Die Weltgesundheitsorganisation prognostiziert bis zum Jahr 2050 einen Anstieg der Zahl der Diabetiker von derzeit 390 Millionen weltweit auf rund 550 Millionen, das sind etwa 10\% der Weltbevölkerung. In Deutschland liegt der Anteil der Personen mit bekanntem Diabetes bei 7\% - etwa 4,5 Millionen Menschen.
\end{abstract}

Zwei Erhebungen des Robert-Koch-Instituts im Rahmen von DEGS (Studie zur Gesundheit Erwachsener in Deutschland) bei jeweils mehr als 7000 zufällig ausgewählten Erwachsenen im Alter von 18-79 Jahren verdeutlichen den Anstieg der Diabetesprävalenz in Deutschland. Demnach ist der Anteil diagnostizierter Diabetiker von 5,6\% bei der Basiserhebung im Jahr 1998 (BGS98) auf 7,2\% bei der 2. Welle in den Jahren 2008-2011 (DEGS-I) gestiegen. Rückläufig ist die Zahl der Personen mit unerkanntem Diabetes und $\mathrm{HbA}_{1 \mathrm{c}} \geq 6,5 \%$; der Anteil sank von $3,8 \%$ auf $2 \%$, so Dr. Christa Scheidt-Nave vom RKI in Berlin.

Die Zahl der Diabetiker in Deutschland (erkannt und unerkannt) wird auf rund 6 Millionen geschätzt - mit gravierenden Konsequenzen. Die Mortalität der Betroffenen ist bei einer Diabetesdauer $>10$ Jahre fast doppelt so hoch wie bei Nichtdiabetikern, sagte PD Dr. Ulrike Rothe aus Dresden. Wird bei einem Mann ein Diabetes im Alter von 40 Jahren diagnostiziert, sinkt seine Lebenserwartung um fast 12 Jahre, bei einer Frau sogar um 14 Jahre. Von den Lebensjahren mit hoher Qualität (QUALY) gehen sogar noch mehr verloren, betonte Rothe.

Als Einflussfaktoren auf die Mortalität von Diabetikern nannte die Epidemiologin den $\mathrm{HbA}_{1 \mathrm{c}}$-Wert, außerdem Rauchen, Alkoholabusus, ländlicher Wohnort, niedriger sozioökonomischer Status sowie Komorbidität wie Gefäßkrankheiten und Depressionen.

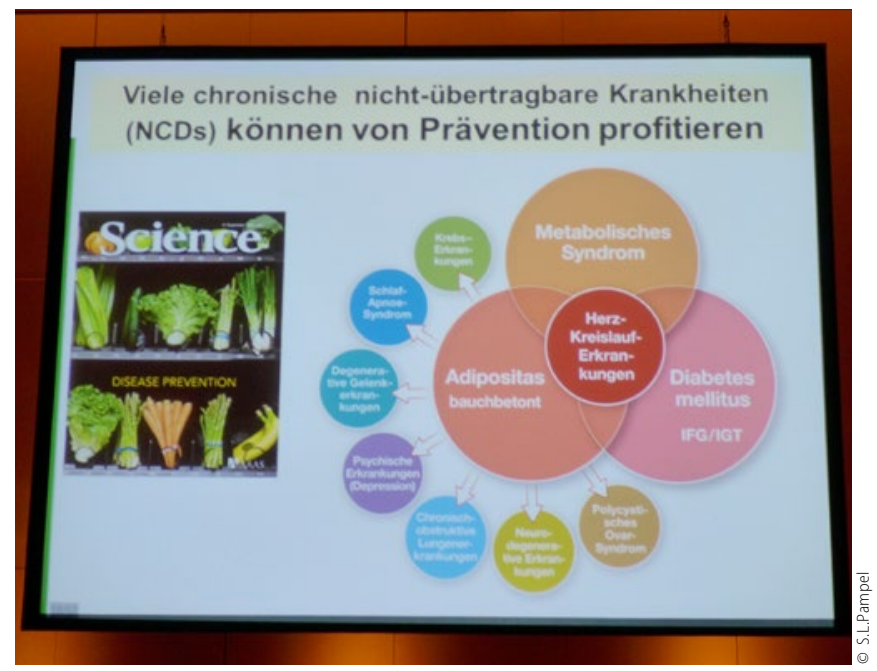

Bei manifestem Diabetes gilt es, alle Optionen zur Prognosebesserung zu nutzen, weil die Schnittmenge zu anderen Krankheiten groß ist. (Quelle Bild: Vortrag Prof. R. Landgraf, DDG-Kongress 2015)

\section{Bessere Versorgungsqualität in Deutschland}

Die Versorgungsqualität bei deutschen Typ-2-Diabetikern hat sich im genannten Zeitraum laut noch unveröffentlichten Daten bei mehr als 300 (BGS98) bzw. 500 (DEGS-I) Studienteilnehmern im Alter von 45-79 Jahren allerdings gebessert. Der Anteil der Patienten mit $\mathrm{HbA}_{1 \mathrm{c}}$-Werten $<7,5 \%$ ist von knapp $50 \%$ auf $80 \%$ gestiegen, berichtete Rothe. Vermehrt vorgenommen werden auch Augenhintergrund-Untersuchungen und Fußchecks. Der Anteil der Raucher liegt konstant bei etwa 20\%, die Rate der Patienten mit Adipositas (Body-Mass-Index > 30) ist von $40 \%$ auf mehr als $55 \%$ gestiegen.

Zwischen der Mortalität und dem BMI von Diabetikern besteht ein U-förmiger Zusammenhang mit einer deutlichen $\mathrm{Ri}$ sikosteigerung bei Personen mit Werten $<20$ und $>35$, sagte Scheidt-Nave. Übergewichtige Patienten hatten allerdings eine höhere Lebenserwartung als normalgewichtige Typ-2-Diabetiker - ein erneuter Hinweis auf das Obesity-Paradox.

Sorge bereitet die starke Gewichtszunahme bei Kindern im Alter $\geq 10$ Jahre. Daten aus dem CrescNet (Raum Leipzig) und der KiGGS (Studie zur Gesundheit von Kindern und Jugendlichen in Deutschland) zufolge hat die Zahl adipöser Kinder (BMI-Perzentile > 97) deutlich auf 6\%-7\% zugenommen, berichtete Prof. Wieland Kiess aus Leipzig. Der Anteil übergewichtiger Kinder (BMIPerzentile > 90-97) im Alter ab 10 Jahren sei bei etwa 15\% stabil. Bei Kindern im Alter bis 6 Jahre ist der Anteil der Übergewichtigen und Fettsüchtigen in Deutschland auch konstant - im Gegensatz zu vielen anderen Ländern wie Saudi-Arabien oder Brasilien.

Als oberstes Ziel zur Prävention nannte Kiess, in der Gesellschaft ein noch stärkeres Bewusstsein für Gesundheit zu schaffen. Bei Kindern und Jugendlichen sollte durch mehr Schulsport und positives Einwirken der Familie der Entwicklung von Übergewicht vorgebeugt werden. Sinnvolle Präventionsansätze seien auch mehr Angebote in Sportvereinen für adipöse Jugendliche und ein metabolisches Screening bei Kinderärzten.

Bei manifestem Diabetes sollten alle Optionen zur Prognoseverbesserung genutzt werden. Ein Verzicht auf orale Antidiabetika wurde mit einer erhöhten Frühsterblichkeit und Sulfonylharnstoffe mit einer erhöhten Mortalität assoziiert, so Rothe. Protektiv wirkten Patientenschulungen und intensivierte Insulintherapie, außerdem optimale Behandlung von weiteren kardiovaskulären Risikofaktoren wie Hypertonie und Fettstoffwechselstörungen.

Roland Fath

Quelle: Diabeteskongress, 50. Jahrestagung der Deutschen Diabetes Gesellschaft (DDG), Berlin, Update-Sitzung Epidemiologie der DDG am 15.5.2015 